\title{
Managing the Balance of Consumption and Reproduction of Marine Resources in the "Coast - Sea” Ecological-Economic System
}

\author{
I. E. Timchenko ${ }^{凶}$, E. M. Igumnova, S. V. Svishchev \\ Marine Hydrophysical Institute of RAS, Sevastopol, Russian Federation \\ 凶timchenko.syst.analysis@mhi-ras.ru
}

Purpose. The work is aimed at constructing an adaptive model of the ecological-economic system of the sea coastal zone intended to control the volume of consumption of marine assimilation and biological resources by the coastal socio-economic system.

Methods and Results. The model based on tracking the integral balance of the rates of marine environment pollution and its purification due to the natural physical and biogeochemical processes and to the environmental actions is proposed. The average multi-year values of the ecosystem variables are used in the applied concept of stationary state of the marine ecosystem. To describe the conditions forming the polluted area in the coastal marine environment, proposed is the algorithm taking into account the ratio between the rate of growth of concentration of the pollutants inflowing from the coastal sources and the rate of their destruction resulting from natural purification of marine environment. Coastal pollution runoff was managed by the penalties imposed on the economic system, which was forced to reduce the generalized product output simultaneously increasing its prime cost. In the marine ecosystem model, the main lower links of the food chain were used: concentrations of phytoplankton, zooplankton and bacterioplankton. Their weighted sum constituted the marine biodiversity index, which was taken as a concentration of bio-resource consumed by the coastal economic system. It was believed that there was an inverse relationship between the biodiversity index and the pollution concentration. Therefore, the maximum permissible pollution concentration was assessed based on the permissible values of the biodiversity index. Along with control of the economic efficiency of marine resource consumption, the model provided for the biodiversity index monitoring so that it did not fall below its permissible vales. In this case, the management agent included in the model, limited production. The model equations are constructed by the method of adaptive balance of causes, which preserved the material balances resulting from the interaction between the environmental and economic processes. The modular equations of the method permitted to use the known stationary state of the ecosystem to obtain the estimates of the influence coefficients. It is shown that the forecasted scenarios provide the compromises between the efficiency of the coastal economic system and the ability of marine ecosystem to restore the consumed assimilation and biological resources.

Conclusions. The proposed model makes it possible to forecast the scenarios of the ecological and economic processes that provide a balance of consumption and reproduction of marine resources.

Keywords: adaptive management model, assimilation and biological resources, coastal economic system, marine ecosystem, adaptive balance of causes, marine pollution, biodiversity index, runoff management agents, forecasted scenarios, influence of sea dynamics

Acknowledgements: the research was carried out within the framework of the state task on theme No. 0827-2018-0004 "Complex interdisciplinary investigations of the oceanology processes conditioning functioning and evolution of the Black and Azov seas' ecosystems of coastal zones". The computational experiments using the model of the ecological-economic system "coast Sevastopol Bay" were performed at the RFBR and Sevastopol government financial support within the framework of scientific project No. 18-47-920001 "Research of the principles of constructing adaptive models of ecological-economic systems and digital information technologies for managing scenarios of sustainable development of natural-economic complexes in the Sevastopol region".

For citation: Timchenko, I.E., Igumnova, E.M. and Svishchev, S.V., 2021. Managing the Balance of Consumption and Reproduction of Marine Resources in the "Coast - Sea" Ecological-Economic System. Physical Oceanography, [e-journal] 28(2), pp. 781-792. doi:10.22449/1573-160X-2021-2-238-254

DOI: 10.22449/1573-160X-2021-2-238-254

(C) I. E. Timchenko, E. M. Igumnova, S. V. Svishchev, 2021

(C) Physical Oceanography, 2021 


\section{Introduction}

Housing construction, the development of industry, agriculture and other forms of intensive development of coastal territories increase the coastal pollution runoff into the sea and stimulate an increase in the consumption of marine resources in the coastal zone of the sea. This leads to a change in the marine environment ecological state. Therefore, one of the main problems of the coastal zone ecological economy is to manage the balance of consumption and reproduction of marine resources [1-3].

A large number of scientific works are devoted to the problem of monitoring the state of marine resources. In the most general formulation, this problem is considered in the models of social ecological and economic systems of the coastal zone of the sea [4-10]. An important aspect of these studies was the assessment of the ability of a complex marine ecosystem to reproduce its equilibrium ecological state under anthropogenic load. In [11-14], a systematic approach to the integral control of the balance of pollution rates and self-purification of the marine environment from pollution supplied to the sea by the coastal economic system was developed. This approach is based on the application of the systemic principle of mutual adaptation of processes in the ecological and economic systems "coast sea” to each other and to external effects. It allows one to control the permissible volumes of resources taken from the marine environment for the economic use. In particular, an adaptive model of the ecological-economic system "coast - sea", in which the management of the intensity of marine biological and assimilation resources consumption depends on ecological parameters - the biodiversity index and the marine environment pollution level [12], was proposed.

The purpose of this study is to construct an adaptive model for managing the ecological and economic processes of consumption and reproduction of the marine environment assimilation and biological resources using a new model for the formation of pollutant concentration in the sea getting from the coastal runoff.

\section{Methods and results}

The marine environment assimilation capacity was defined as the maximum possible rate of self-purification from pollution, at which irreversible changes do not begin in the marine ecosystem, preventing its return to an equilibrium stationary state. In the general case, the resource capacity was represented by the limiting value of consumption and recovery rates of the concentration of a particular type of resources, when these rates are still equal, but are already at the upper limit of their possible values.

In order to construct a dynamic model of control over the consumption and reproduction of marine resources, quantitative measures of the consumed resource per unit volume of the marine environment have been introduced: the biodiversity index $(B D)$ for the biological resource and the pollution level index $(P L)$ for the assimilation resource. A multitude of positive and negative factors that form the scenarios of changes in the concentration of each type of resources have 
determined the scheme of cause-effect relationships of the marine ecosystem model. The resource concentration was expressed as a weighted sum of the concentrations of those substances that are included in the material balance equation that forms a given type of resource in a specific environment volume. The dynamic ecosystem model consists of differential equations of adaptive balance of causes method [11], preserving the indicated material balances. Therefore, the research objectives include the development of a method for determining the effect coefficients in the equations of the marine ecosystem adaptive model and carrying out computational experiments to test the adequacy of the proposed model to the existing ideas about the coastal pollution runoff impact on the scenarios of ecological and economic processes in the sea coastal zone.

The general property of natural ecosystems adaptation to external effects is known. It is expressed in the fact that the system tends to maintain a stable equilibrium state, in which a balance of forces (effects) takes place and the dynamic mode "action equals counteraction" is maintained. In adaptive models of marine ecosystems, the equality of the rates of resource consumption and its reproduction is provided by a negative feedback between the amount of the consumed resource and its reproduction. A decrease in the resource concentration causes (within certain limits) an increase in the rate of its reproduction to a level that compensates for the increased rate of the resource consumption.

\section{Integral model for the formation of concentration variance scenario of marine environment pollutants}

Now we are to consider one of the possible variants of an integral model for the formation of a concentration variance scenario of the coastal marine environment pollutants, which come from a distributed coastal source. In this case, we are talking about the consumption of the marine environment assimilative resource and its assimilation capacity. The concentration variations are the result of two opposite processes - the accumulation of pollutants and self-purification from them due to biochemical destruction. For the sake of simplicity, it can be assumed that the pollution level index is represented by $P L$ concentration of one particular pollutant type.

We assume that a pollutant flux with $P L_{A}$ concentration begins to get into a marine environment limited volume with a constant (background) concentration of pollutants $P L_{n}$. Then scenarios of pollutant concentration variation in the volume of the environment will begin to form according to a certain law. We will assume that this law is determined by two main factors: mixing, which evenly distributes pollutants throughout the entire volume of medium, and biochemical decomposition reactions of pollutants occurring in the marine ecosystem. We also accept $P L_{n}<<P L_{A}$, i.e. the background concentration of $P L_{n}$ pollutants in the sea is much less than the concentration of pollutants in the runoffs $P L_{A}$ entering the sea. These assumptions mean that the concentration of pollutants in the marine environment varies within $P L_{n} \leq P L \leq P L_{A}$. 
Without taking into account biochemical self-purification reactions that decompose the pollution, the concentration $P L^{(+)}(t)$ will increase due to marine environment dynamics from an initial value $P L_{n}$ to $P L_{A}$ value according to the exponential law with saturation:

$$
P L^{(+)}(t)=P L_{n}+\left[P L_{A}-P L_{n}\right][1-\exp (-\alpha t)]
$$

The exponent $\alpha$ determines the rate of pollutant concentration increase in the sea under effect of molecular and turbulent diffusion, i.e., the rate of consumption by the pollution source (coastal social-economic system) of the marine environment assimilation resource.

Along with this process, the biochemical self-purification reactions of the ecosystem will strive to return the pollutant concentration to the equilibrium background value $P L_{n}$, decreasing it from $P L^{+}(t)$ to $P L_{n}$. In this case, the reproduction rate of the assimilation resource will determine the parameter $\beta$ in the formula

$$
P L(t)=P L^{(+)}(t)+\left[P L_{n}-P L^{(+)}(t)\right][1-\exp (-\beta t)]
$$

Taking into account the variable power of the pollution source, the concentration of pollutants is a time function $P L_{A}(t)$. Substituting in (2) instead of $P L^{(+)}(t)$ its expression (1), we obtain the general law of in the pollutant concentration variations in the sea under effect of both dynamic and biochemical factors:

$$
\left.P L(t)=P L_{n}+\left[P L_{A}(t)-P L_{n}\right][1-\exp (-\alpha t)] \exp (-\beta t)\right]
$$

Thus, the dynamic balance of the environment pollution and self-purification rates is determined by the parameters $\alpha$ and $\beta$, which set the rates of consumption and reproduction of the assimilation resource. At a constant marine environment pollution source $P L_{A}$, differentiating the last equality and equating the derivative to zero, we find a condition linking the time of reaching the maximum concentration of pollutants with the assimilation resource consumption and reproduction rates:

$$
t^{*}=\frac{1}{\alpha} \ln \frac{\alpha+\beta}{\beta} .
$$

In the ecosystem steady state, when the assimilation capacity consumption and reproduction rates coincide $(\alpha=\beta)$, the time for reaching the maximum concentration pollutants $P L_{A}$ is $t^{*}=\alpha^{-1} \ln 2$ arbitrary units. 
The proposed mechanism for the formation of concentration variance scenario of marine environment pollutants should exist within the ecosystem capabilities to resist the pollution increase when the concentration of pollutants does not exceed the approximate permissible level $P L^{*}$. This value is critical by definition, since at it the reproduction rate of marine biodiversity index begins to lag behind the rate of its consumption. When the pollution level exceeds $P L^{*}$ value, the biochemical reactions of pollution destruction begin to weaken, which can cause the degradation of the marine ecosystem. It is natural to assume that at $P L(t) \geq P L^{*}$ the rate of self-purification from contamination will decrease. In order to take this circumstance into account, it is necessary to include in formula (2) a control agent $\beta(\tau)$, which will slow down the rate of biochemical selfpurification by changing the value of $\beta$ coefficient when $P L>P L^{*}$ :

$$
\begin{gathered}
P L(t)=P L_{n}+\left[P L_{A}(t)-P L_{n}\right][1-\exp (-\alpha t)] \exp [-\beta(\tau) t], \\
\beta(\tau)=\beta^{*} I F\left[P L<P L^{*} ; 1 ; \exp (-\gamma \tau)\right],
\end{gathered}
$$

where $\beta^{*}$ is a parameter in the ecosystem stationary state.

Thus, the dynamics of the pollution level is limited to segments of $0 \leq P L(t) \leq P L^{*} \leq P L_{A}$ values, the limits of which depend on the ratio of consumption and reproduction rates of the marine environment assimilation resource.

\section{Self-purification rate control in the pollutant concentration equations}

Expression (3) can be used as an external effect source in the right side of the dynamic equation for the pollutant concentration, constructed by the adaptive balance of causes method [11]. In the adaptive equation, the variation rate in the pollutant concentration is associated with a negative second-order feedback $[13,14]$ with the concentration itself:

$$
\frac{d P L}{d t}=2 r_{P L} P L\left\{C_{P L}-\left[P L-\left(A_{P L}-C_{P L}\right)\right]\right\},
$$

where $r_{P L}$ is a relative rate of $P L$ concentration variation; $A_{P L}$ is an external source deviating this concentration from the stationary (mean) value $C_{P L}$.

We will assume that at the initial time moment the concentration of pollutants has a stationary background value $P L_{n}=C_{P L}$, which corresponds to the mean longterm values $\left(C_{i}\right)$ of the marine ecosystem model variables $\left(u_{i}\right)$. Then from the formula (3) the external pollution source $A_{P L}$ in the equation (4) will be determined and it will take the following form: 


$$
\left.\frac{d P L}{d t}=2 r_{P L} P L\left\langle C_{P L}-\left\{P L-\left[P L_{A}(t)-C_{P L}\right][1-\exp (-\alpha t)] \exp [-\beta(\tau) t)\right]\right\}\right\rangle
$$

To test the proposed model for the scenario formation of the pollutant concentration variance, computational experiments were carried out. Equation (8) is represented in finite differences with sampling interval in time $\Delta t$. For simplifying the solution algorithm, an additional condition $2 r_{P L} C_{P L} \Delta t=1$, which can always be satisfied by choosing a time interval $\Delta t$, was adopted. As a result, the following iterative process was proposed:

$$
P L^{k+1}=2 P L^{k} 1-\left(2 C_{P L}\right)^{-1}\left\{P L^{k}-\left[P L_{A}^{k}-C_{P L}\right][1-\exp (-\alpha \Delta t k)] \exp [-\beta(\Delta t l) \Delta t k]\right\} .
$$

In Fig. 1 the scenarios of concentration variation of pollutants obtained under various conditions are given. As follows from formula (3), with constant coefficients $\alpha$ and $\beta$, these scenarios have a single maximum, the position of which is determined by expression (4). Concentrations of pollutants should tend to a stationary value $P L_{n}=C_{P L}$ at $t \rightarrow \infty$.
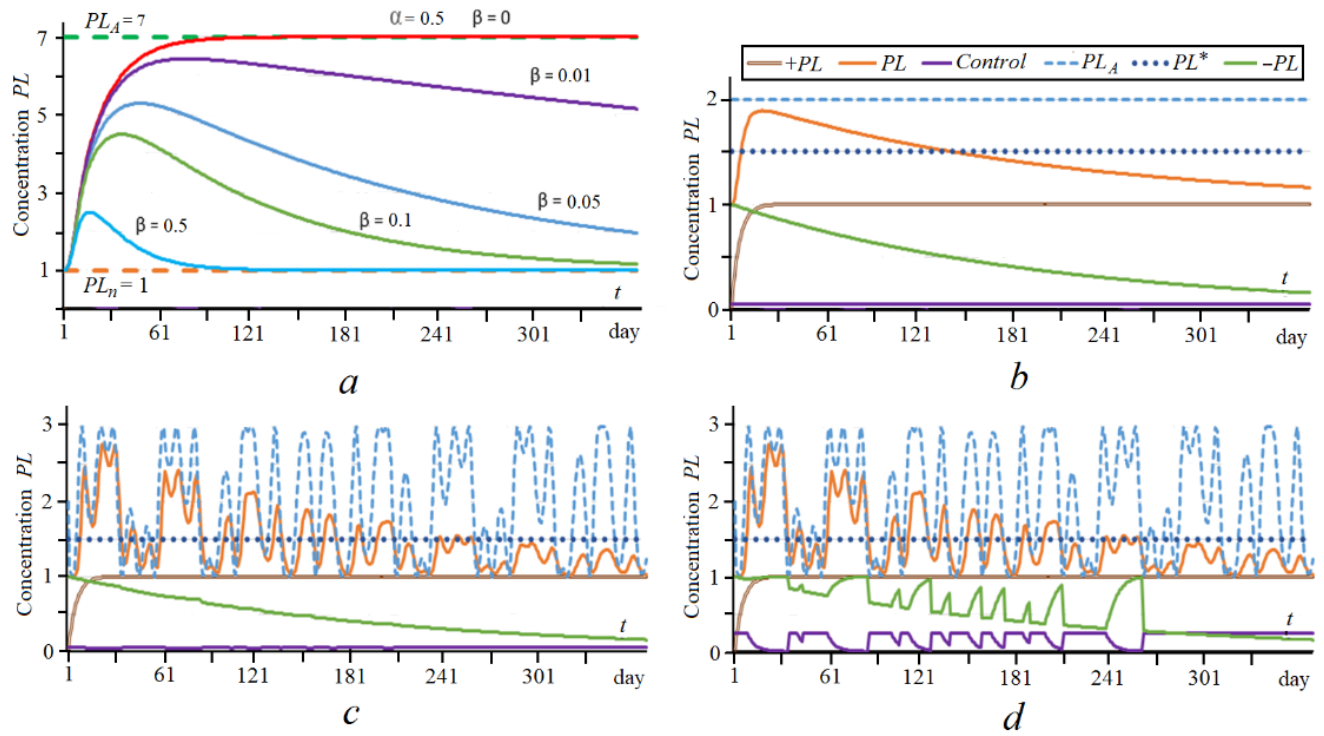

F i g. 1. Change of the pollutants $P L$ concentration in the marine environment with constant $(a, b)$ and variable $(c, d)$ sources of pollution $P L_{A}$ by the scenarios according to which: the rates of the environment self-purification are different $(a)$; control of the environment self-purification rate (graph Control) is turned off $(b, c)$; control is on $(d)$

This conclusion is confirmed by a family of scenarios (Fig. 1, a) obtained at different rates of the medium self-purification, when the stationary value of pollutant concentration, the source intensity, and the rate of the concentration increase in 
the sea are constant. Fig. 1, $b-d$ demonstrates the graphs of functions (forming the scenarios of the pollutant concentration in the sea), which have the following notations: $+P L$ is an increase in concentration according to formula (1), $-P L$ is a decrease in concentration according to formula (2), Control is an ecosystem selfpurification rate control by formula (6). In order to simulate random variations in the pollutant concentration in the sea, a random function $P L_{A}$, depicted in Fig. 1, $c$, $d$, was applied. Comparing the scenarios $P L$ in Fig. 1, $a, b$, it follows that controlling the rate of the ecosystem self-purification led to an increase in pollution during periods of time when $P L(t) \geq P L^{*}$. Thus, computational experiments have confirmed the correctness of the concept of the marine environment pollution level formation, laid down in the model (8) - (9).

\section{Adaptive model of the "cost - sea” ecological-economic system management}

A conceptual model for managing the balance of consumption and reproduction of marine environment assimilative and biological resources is given in Fig. 2. The model consists of two interconnected subsystems - economic ("coast") and ecological ("sea”). Management of the "coast - sea" system is carried out by the agents $A G_{V}$ and $A G_{B D}$, which manage the economic profitability of the marine environment assimilative and bioresource capacities use by the coastal social-economic system [12], taking into account the proposed above model of the sea pollution level formation.

The main condition for managing the system is that the biodiversity index $B D$ does not fall below the estimated permissible value $B D^{*}$, which is taken as the "normal" ecological state of the marine environment. It is assumed that there is an inverse functional relationship between the biodiversity index $B D^{*}$ and the estimated permissible pollution level in the sea $P L^{*}$. The integral balance of accumulated and assimilated pollution $S$ over the time interval $(0, t)$ is controlled by the agent of the marine environment resource capacity $R(t)$ :

$$
R(t)=I F\left(\begin{array}{l}
P L<P L^{*} ; 1 ; I F\left\{T X_{\mathrm{acc}}<T X_{\mathrm{acc}}^{*} ; \exp \left(-\alpha_{R} \tau\right)\right. \\
R(t)+[1-R(t)]\left[1-\exp \left(-\beta_{R} \tau\right]\right\}
\end{array}\right)
$$

The logical management operator (10) monitors the excess of the pollution level $P L^{*}$ with regard to the difference in the rates of pollutant accumulation in the block $S_{\text {acc }}$ and their assimilation in the block $S_{\text {ass }}$ (Fig. 2). The agent for marine environment purification from pollution activates the runoff purification mode when the value of the treatment fund $T X_{\text {acc }}$ exceeds the $T X_{\text {acc }}^{*}$ value. 


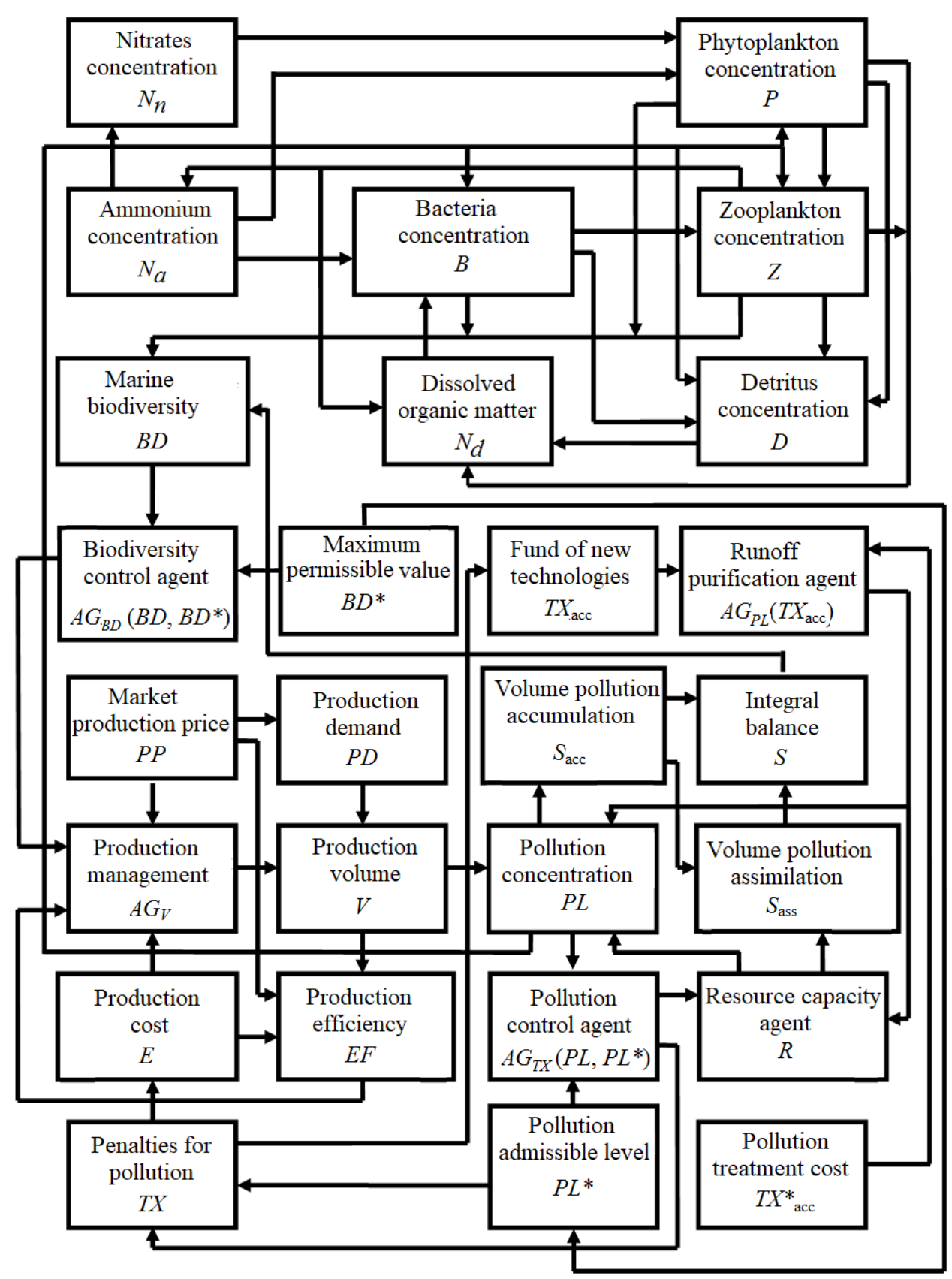

F i g. 2. Scheme of cause-effect relations in the adaptive model of managing the balance of consumption and reproduction of the assimilation and biological resources of marine environment

The system of the model equations describing the relations between the elements of $u_{i}$ ecosystem demonstrated in Fig. 2, consists of $n$ modular equations of the adaptive balance of causes method [12-14], which have the following general form: 


$$
\frac{d u_{i}}{d t}=2 r_{i} u_{i}\left\{C_{i}-\left[u_{i}-\sum_{k=1, k \neq i}^{m} a_{i k}\left(u_{k}-C_{k}\right)+\sum_{l=m+1, l \neq i}^{n} a_{i l}\left(u_{l}-C_{l}\right)-A_{i}\right]\right\}
$$

For estimating the effect coefficients in (11), the assumption that the average long-term values $C_{i}$ of all the model variables are known and the variability intervals of variables have the form $0 \leq u_{i} \leq 2 C_{i}$ was used. Equations (11) describe the processes of adaptation of the "coast - sea" system $u_{i}$ products to its resources - positive $\left(u_{k}\right)$ and negative $\left(u_{l}\right)$. The adaptation processes reflect the system model reaction to the deviations of its variables from the mean (stationary) state under the influence of external effects. The effect coefficients $a_{i k}$ and $a_{i l}$ show what increase in product concentration gives an increase in the resource affecting it: $u_{i}-C_{i}=a_{i j}\left(u_{j}-C_{j}\right)$. The assumption of a known stationary state of the "coast - sea" system allows one to introduce the estimates of the effect coefficients for this state and extend them to arbitrary times with corrections for the current values of the variables.

In order to obtain such estimates of $a_{i j}$ coefficients, the processes $u_{j}$, affecting the process $u_{i}$, are reduced to the dimension of this process by the following transformation: $u_{i}=a_{i j}^{\prime} C_{i} C_{j}^{-1} u_{j}$. Next, the normalization of dimensionless factors $a_{i j}^{\prime}$, taking into account the current values of all affecting processes, is carried out. Then the system of equations (11) can be represented as

$$
\begin{aligned}
& \frac{d u_{i}}{d t}=2 r_{i} u_{i}\left\{C_{i}-\left[u_{i}-0,5 \sum_{j=1, j \neq i}^{m} C_{j}^{-1} u_{j}\left(\sum_{k=1, j \neq i}^{m} C_{k}^{-1} u_{k}\right)^{-1} C_{i} C_{k}^{-1}\left(u_{k}-C_{k}\right)+\right.\right. \\
& \left.\left.+0,5 \sum_{j=m+1, j \neq i}^{n-m} C_{j}^{-1} u_{j}\left(\sum_{l=m+1, j \neq i}^{n-m} C_{l}^{-1} u_{l}\right)^{-1} C_{i} C_{l}^{-1}\left(u_{l}-C_{l}\right)-A_{i}\right]\right\} .
\end{aligned}
$$

The equations for each variable of the ecological-economic system model, taking into account the intrasystem and external effects shown in Fig. 2, are as follows:

$$
\begin{aligned}
& \frac{d P P}{d t}=2 r_{P P} P P\left\{C_{P P}-\left[P P-C_{P P}\left(a_{21} \mathrm{NO}_{3}^{\prime}+a_{23} \mathrm{NH}_{4}^{\prime}-a_{25}-a_{26} D O N^{\prime}-\right.\right.\right. \\
& \left.\left.\left.-a_{27} D^{\prime}-a_{28} P L_{Z}^{\prime}\right)\right]\right\}, \\
& \frac{d Z}{d t}=2 r_{Z} Z\left\{C_{Z}-\left[Z-C_{Z}\left(a_{21} P^{\prime}+a_{23} B^{\prime}-a_{25} \mathrm{NH}_{4}^{\prime}-a_{26} D O N^{\prime}-a_{27} D^{\prime}-a_{28} P L_{Z}^{\prime}\right)\right]\right\}, \\
& \frac{d B}{d t}=2 r_{B} B\left\{C_{B}-\left[B-C_{B}\left(a_{36} D O N^{\prime}-a_{32} Z^{\prime}-a_{35} \mathrm{NH}_{4}^{\prime}-a_{38} P L_{B}^{\prime}\right)\right]\right\}, \\
& \frac{d \mathrm{NO}_{3}}{d t}=2 r_{\mathrm{NO}_{3}} \mathrm{NO}_{3}\left\{C_{\mathrm{NO}_{3}}-\left[\mathrm{NO}_{3}-C_{\mathrm{NO}_{3}}\left(a_{45} \mathrm{NH}_{4}^{\prime}+a_{46} D O N^{\prime}+a_{48} P L_{\mathrm{NO}_{3}}^{\prime}\right)\right]\right\},
\end{aligned}
$$




$$
\begin{aligned}
& \frac{d \mathrm{NH}_{4}}{d t}=2 r_{\mathrm{NH}_{4}} \mathrm{NH}_{4}\left\{C_{\mathrm{NH}_{4}}-\left[\mathrm{NH}_{4}-C_{\mathrm{NH}_{4}}\left(a_{52} Z^{\prime}+a_{53} B^{\prime}+a_{56} D O N^{\prime}-a_{51} P^{\prime}-\right.\right.\right. \\
& \left.\left.\left.-a_{54} \mathrm{NO}_{3}^{\prime}+a_{58} P L_{\mathrm{NH}_{4}}^{\prime}\right)\right]\right\}, \\
& \frac{d D O N}{d t}=2 r_{D O N} D O N\left\{C_{D O N}-\left[D O N-C_{D O N}\left(a_{61} P^{\prime}+a_{62} Z^{\prime}+a_{67} D^{\prime}-a_{63} B^{\prime}-\right.\right.\right. \\
& \left.\left.\left.-a_{64} \mathrm{NO}_{3}^{\prime}-a_{65} \mathrm{NH}_{4}^{\prime}+a_{68} P L_{D O N}^{\prime}\right)\right]\right\}, \\
& \frac{d B D}{d t}=2 r_{B D} B D\left\{C_{B D}-\left[B D-A G_{B D}(P P, Z P, B)\right]\right\},
\end{aligned}
$$$$
A G_{B D}(P P, Z P, B)=C_{B D} \arg \min \left(C_{P P}^{-1} P P ; C_{Z P}^{-1} Z P ; C_{B}^{-1} B\right),
$$$$
\frac{d D}{d t}=2 r_{D} D\left\{C_{D}-\left[D-C_{D}\left(a_{71} P^{\prime}+a_{72} Z^{\prime}-a_{76} D O N^{\prime}+a_{78} P L_{D}^{\prime}\right)\right]\right\},
$$$$
\frac{d P L}{d t}=2 r_{P L} P L\left\langle C_{P L}-\left\{P L-\left[C_{P L}\left(a_{P L / V} C_{V}^{-1} V^{\prime}\right)-\right.\right.\right.
$$$$
\left.\left.\left.\left.-C_{P L}\right][1-\exp (-\alpha t)] \exp [-\beta(\tau) t)\right]+A G\left[T X_{\text {acc }}, T X_{\text {acc }}^{*}\right]\right\}\right\rangle \text {, }
$$$$
A G\left(T X_{\text {acc }}, T X_{\text {acc }}^{*}\right)=I F\left\{T X_{\text {acc }},<T X_{\text {acc }}^{*} ; 0 ; C_{P L}[1-\exp (-\delta \tau)]\right\},
$$$$
T X_{\mathrm{acc}}(t)=\int_{0}^{t} T X(\tau) d \tau,
$$$$
\frac{d P D}{d t}=2 r_{D P} P D\left[C_{D P}-\left(P D+a_{D P / P P} P P^{\prime}\right)\right]
$$

$$
\begin{gathered}
\frac{d V}{d t}=2 r_{V} V\left\{C_{V}-\left[V-a_{V / D P} P D^{\prime}+A G_{V}(P, E)+A G_{V}\left(B D, B D^{*}\right)\right]\right\}, \\
A G_{V}(P, E)=I F\left\{P>E ; 0 ; C_{V}\left[1-\exp \left(-\alpha_{V / P} \tau\right)\right]\right\}, \\
A G_{V}\left(B D, B D^{*}\right)=I F\left\{B D>B D^{*} ; 0 ; C_{V}\left[1-\exp \left(-\alpha_{V / B D} \tau\right)\right]\right\}, \\
\frac{d T X}{d t}=2 r_{T X} T X\left\{C_{T X}-\left[T X-C_{T X} C_{P L}^{-1} P L^{\prime}-A G_{T X}\left(P L, P L^{*}\right)-A G_{T X}\left(B D, B D^{*}\right)-T X^{*}\right]\right\}, \\
A G_{T X}\left(P L, P L^{*}\right)=C_{T X} I F\left\{P L<P L^{*} ; 0 ; k_{T X 1}\left[1-\exp \left(-\alpha_{T X} \tau\right)\right]\right\}, \\
A G_{T X}\left(B D, B D^{*}\right)=C_{T X} I F\left\{B D>B D^{*} ; 0 ; k_{T X 2}\left[1-\exp \left(-\alpha_{T X} \tau\right)\right]\right\} .
\end{gathered}
$$

The dashes in equations (13) denote the deviations of the variables from their mean values, and the asterisks denote the given values. The agent $A G_{B D}(P P, Z P, B)$ in the equation for the biodiversity index limits this index by the value of the minimum living objects concentration in the ecosystem. The efficiency of the marine environment resource consumption is assessed 
integrally by the ratio of the incomes of economic system $I_{\text {acc }}(t)$ and its costs $E_{\text {acc }}(t)$ for the time period $(0, t)$ :

$$
I_{\text {acc }}=\int_{0}^{t} P^{*} V(t) d t, \quad E_{\mathrm{acc}}=\int_{0}^{t} E(t) V(t) d t, \quad E F=\ln \frac{1+I_{\mathrm{acc}}}{1+E_{\mathrm{acc}}} .
$$

The effect coefficients in the equations of the marine ecosystem were calculated using the normalized ratios of the mean values, as shown in the system of equations (13).

\section{Managing the process of marine environment purification from the pollutants in the adaptive model of the "coast - Sevastopol Bay" ecological-economic system}

The integral management of the marine environment pollution level by the coastal runoff into the Sevastopol Bay is considered in [12-14] without taking into account the environment self-purification model proposed in this study in the equation for the pollutant concentration. In order to construct the scenarios of ecological and economic processes, we use the mean values of the concentrations (in $\mathrm{mgN} / \mathrm{m}^{3}$ ) of the modeled substances in the bay ecosystem known from longterm observations, which were taken as the ecosystem stationary state: phytoplankton - 37.74, zooplankton - 18.87, bacterioplankton -12.0 , nitrates 42.35, ammonium - 5.60, dissolved organic forms of nitrogen - 18.0, detritus 15.0. We assume that the coastal economic subsystem produces a generalized product and directs into the bay the pollution flux, proportional to the production volume. The system of equations is written down in finite differences for constructing iterative cycles similar to (9). The estimates of the effect coefficients of the bay ecosystem equations are calculated by the normalized ratios of the average values of the variables by the formulas used in the system of equations (12). A system of dimensionless economic variables, reduced to a single scale $(0,10)$, is applied. The calculations are performed for one year (365 time steps).

In Fig. 3 the scenarios of ecological and economic processes in the "coast Sevastopol Bay” system are given. They were reconstructed by the model (12) (14), when the bay was not polluted by the coastal economic subsystem, and, consequently, there was no control and management of the pollution level.

Output of products $V$ repeats the predetermined seasonal variation of demand for it $P D$ (Fig. 3, a). The rates of consumption and reproduction of the bay resources are equal, and the values of the biodiversity index $B D$ are significantly higher than the permissible level $B D^{*}$ (Fig. 3, b). Despite the absence of pollution in the bay, the system continues to impose a pollution penalty proportional to production volume. It is considered as an environmental tax that replenishes the $T X_{\text {acc }}$ fund for the runoff purification from pollution (Fig. 3, c). The production profitability reaches a stable maximum (Fig. 3, d), and the bay ecosystem is in a stable stationary state (Fig. $3, e, f$ ). 


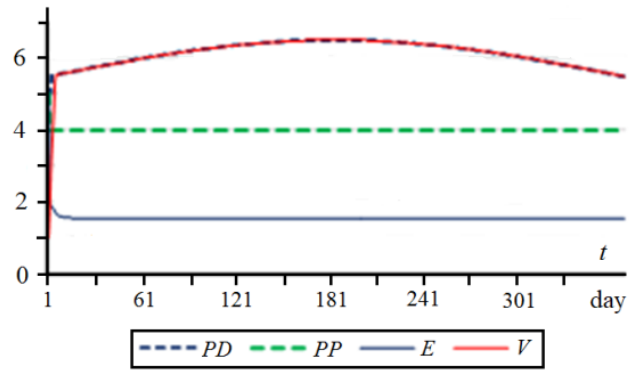

$a$
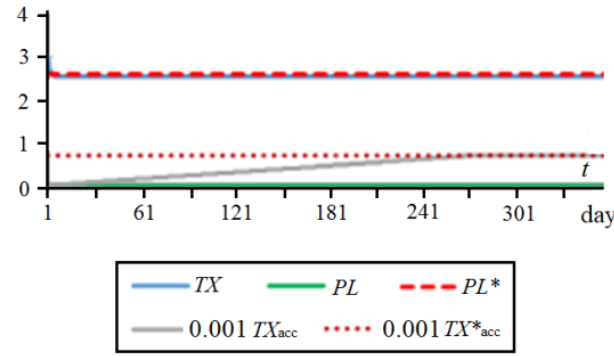

$c$

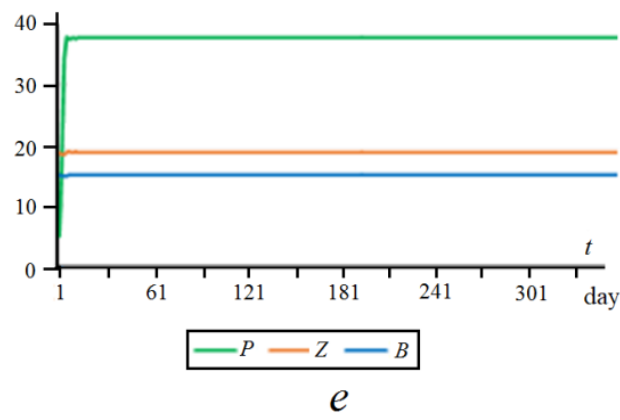

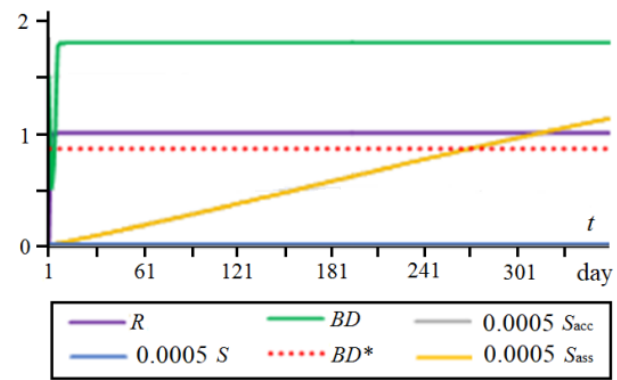

$b$
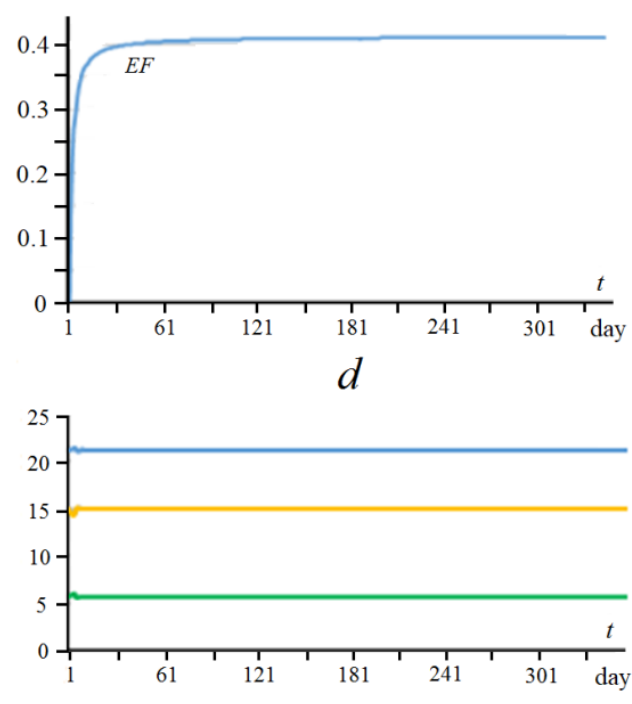

$-0.5 N_{n}-N_{a}-N_{d}-D$
$f$

F i g. 3. Scenarios of the processes in the ecological-economic system "coast - Sevastopol Bay" in case the marine pollution is absent

In the next experiment, the mechanisms for managing the consumption and reproduction balance of the marine environment assimilative and biological resources are tested according to the criteria of pollution level and the biodiversity index. For this purpose, the mechanisms for monitoring and managing the levels of pollution and biodiversity of the bay marine environment are turned on, but without taking into account the dynamics of the marine environment. The experimental results are shown in Fig. 4. The production volume $V$ and the pollution flux related to it are limited in the first part of the experiment (Fig. 4, $a, c$ ) for a number of reasons. The first limitation occurs at the $75^{\text {th }}$ step, when the biodiversity index falls below $B D *$ value (Fig. 4, b); the - second at the $125^{\text {th }}$ step, when $T X$ penalties reach the maximum values (Fig. 4, c) and the production cost $E$ exceeds the cost of the generalized product $P P$ (Fig. 4, a). Subsequent restrictions are due to the drop in the biodiversity index value and with the reaction of the of the resource capacity agent $R(t)$ to this (Fig. 4, $b$ ). 


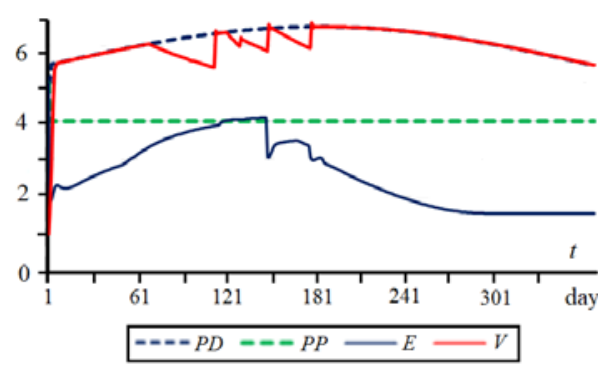

$a$
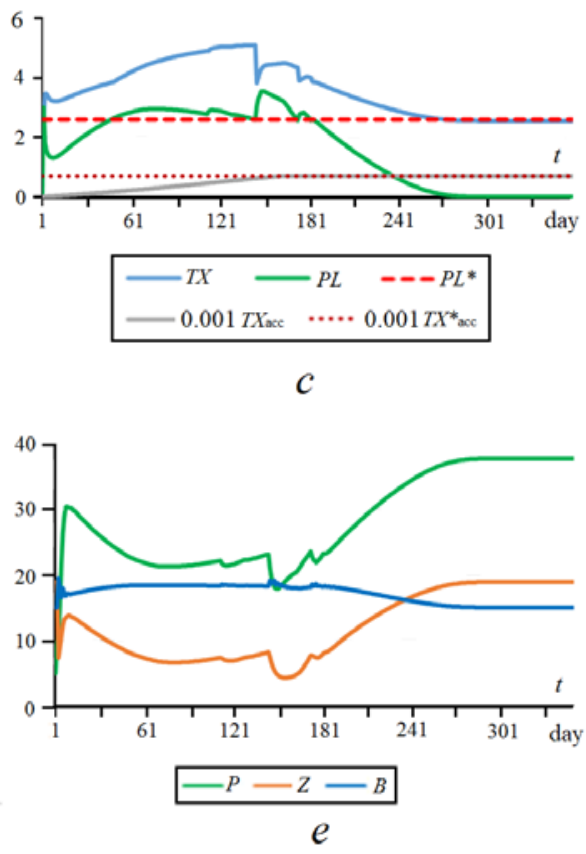

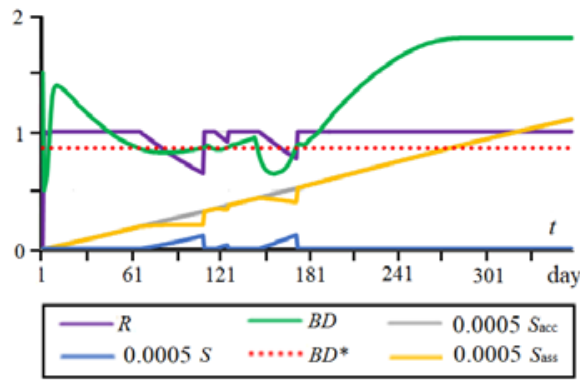

$b$
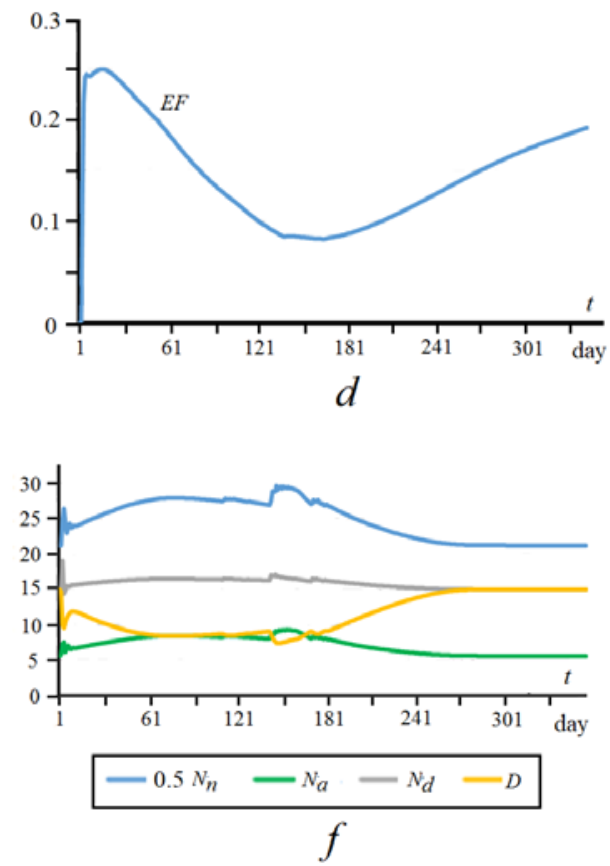

F i g. 4. Managing the balance of consumption and reproduction of marine resources in the "coast sea" ecological-economic system based on the criteria of the pollution level and the biodiversity index with no account of the marine environment dynamics. The runoff purification mode is on at the $148^{\text {th }}$ step of calculations

In the second part of the experiment, the funds accumulated in $T X_{\text {acc }}$ runoff purification fund were sufficient to start the purification mode (Fig. 4, c), and $A G_{P L}$ ( $T X_{\text {acc }}$ ) agent turned on this mode at the $148^{\text {th }}$ step. As a result, the pollutant concentration decreases sharply and, in accordance with formulas (6) and (8), the self-purification rate increases. In the subsequent period of time, the development processes acquire positive dynamics, since the production output increases to the of seasonal demand value.

Due to the reduction of fines (Fig. 4, c), the production cost decreases (Fig. 4, a), and its profitability is steadily growing (Fig. 4, d). As a result, the ecosystem model biochemical variables take their stationary values (Fig. 4, $e, f$ ). 


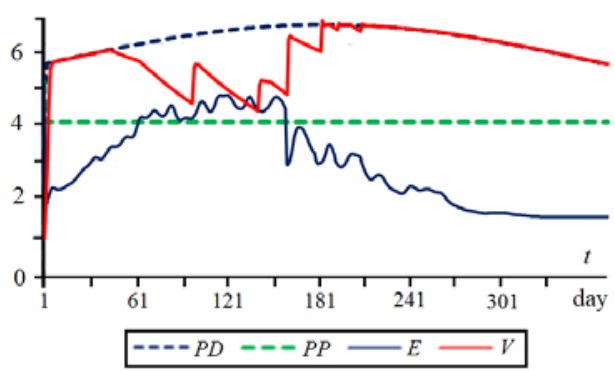

$a$

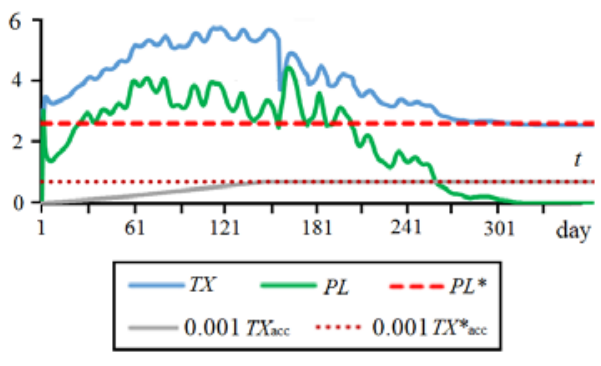

$c$

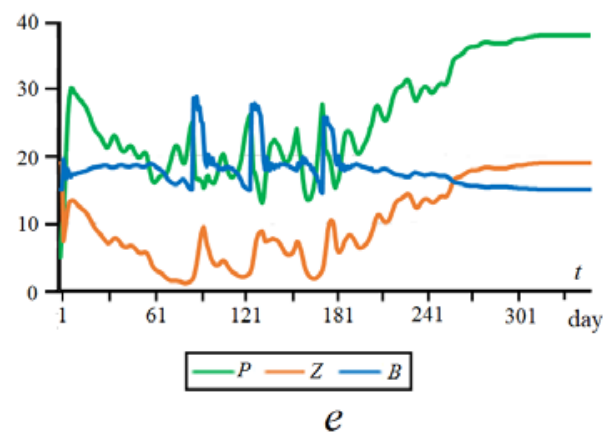

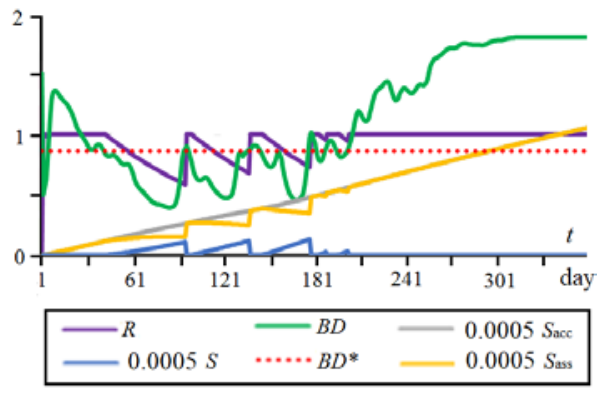

$b$
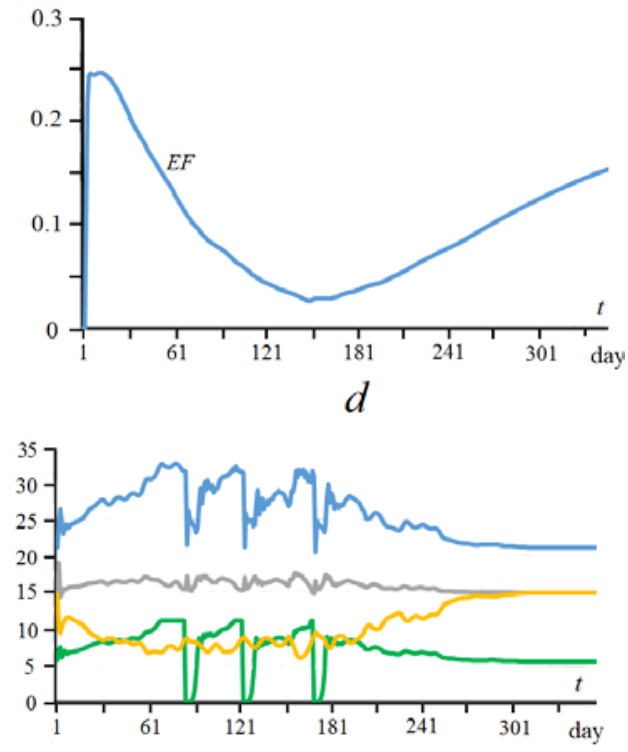

$\frac{\square-0.5 N_{n}-N_{a}-N_{d}-D}{f}$

F i g. 5. Influence of marine environment dynamics on the balance of consumption and reproduction of its assimilation and biological resources in the "coast - sea" ecological-economic system based on the criteria of the pollution level and the biodiversity index

The influence of the marine environment dynamics on the scenarios of Fig. 4 was considered during the experiment, the results of which are demonstrated in Fig. 5. The main influence on the pollutant concentration in the sea upper layer is exerted by the transfer and diffusion of water masses, as well as wave processes caused by random fluctuations in the wind velocity module. In order to simulate the wind effect on the pollutant concentration, a random function $P L_{a}$ was added to the equation for $P L$ system of equations (13), which was used earlier in the model (8) for the formation of pollutant concentration variation scenario (Fig. 1, $c, d$ ). 
From a comparison of the scenarios of ecological and economic processes in Fig. 4, $c$ and 5, $c$ it follows that the duration of the periods and the values of the excess concentration of pollutants in the approximate permissible level of pollution $P L^{*}$ become significantly higher. In accordance with the adopted model (5) - (6), the marine environment self-purification rates at $P L \geq P L^{*}$ slow down. As a result, the fines and production costs increase, which leads to a limitation in output and a drop in its profitability (Fig. 5, $a, c, d$ ). The dynamics of pollutant concentration significantly complicates the scenarios of biochemical processes in the marine ecosystem (Fig. 5, e, f). This entails variations in $B D$ biodiversity index. Note that, by definition, the biodiversity agent $A G_{B D}(P P, Z P, B)$ in the system of equations (13) reacts to the minimum values of the biological variables of the model managed by it. Since the scenario of zooplankton concentration variation (Fig. $5, e$ ) has the minimum values, the biodiversity index scenario is formed under the effect of this concentration (Fig. 5, b).

\section{Conclusion}

The purpose of this study was to develop an adaptive model for managing the ecological and economic processes of consumption and reproduction of the marine environment assimilation and biological resources. This goal was achieved by applying an additional mechanism for managing the rate of marine environment purification from pollution, which reacted to changes in its ecological state. When the pollutant concentration exceeded the value of the indicative pollution permissible level, and the biodiversity index fell below its permissible level, the special agent slowed down the rate of environment selfpurification from pollution getting from the coastal runoff. The ecosystem transition into a mode of imbalance in the consumption and reproduction of marine environment assimilation and biological resources is presented. Verification of the proposed mechanisms for coastal runoff management by analyzing the predicted scenarios of pollution level and the environmental biodiversity index shows that they provide a compromise between the coastal economic system profitability and the marine ecosystem ability to recover consumed assimilative and biological resources.

\section{REFERENCES}

1. Daly, H. and Farley, J., 2010. Ecological Economics: Principles and Applications. Washington, DC: Island Press, 544 p.

2. Halpern, B.S., Walbridge, S., Selkoe, K.A., Kappel, C.V., Micheli, F., D'Agrosa, C., Bruno, J.F., Casey, K.S., Ebert, C. [et al.], 2008. A Global Map of Human Impact on Marine Ecosystems. Science, 319(5865), pp. 948-952. doi:10.1126/science.1149345

3. Börger, T., Beaumont, N.J., Pendleton, L., Boyle, K.J., Cooper, P., Fletcher, S., Haab, T., Hanemann, M., Hooper, T.L. [et al], 2014. Incorporating Ecosystem Services in Marine Planning: The Role of Valuation. Marine Policy, 46, pp. 161-170. https://doi.org/10.1016/j.marpol.2014.01.019

4. Costanza, R., de Groot, R., Sutton, P., van der Ploeg, S., Anderson, S.J., Kubiszewski, I., Farber, S. and Turner, R.K., 2014. Changes in the Global Value of Ecosystem Services. Global Environmental Change, 26, pp. 152-158. http://dx.doi.org/10.1016/j.gloenvcha.2014.04.002 
5. Long, R.D., Charles, A. and Stephenson, R.L., 2015. Key Principles of Marine EcosystemBased Management. Marine Policy, 57, pp. 53-60. http://dx.doi.org/10.1016/j.marpol.2015.01.013

6. Samhouri, J.F. and Levin, P.S., 2012. Linking Land- and Sea-Based Activities to Risk in Coastal Ecosystems. Biological Conservation, 145(1), pp. 118-129. https://doi.org/10.1016/j.biocon.2011.10.021

7. Crépin, A.-S., Norberg, J. and Mäler, K.-G., 2011. Coupled Economic-Ecological Systems with Slow and Fast Dynamics - Modelling and Analysis Method. Ecological Economics, 70(8), pp. 1448-1458. https://doi.org/10.1016/j.ecolecon.2011.02.004

8. Costanza, R., Voinov, A., Boumans, R., Maxwell, T., Villa, F., Wainger, L. and Voinov, H., 2002. Integrated Ecological Economic Modeling of the Patuxent River Watershed, Maryland. Ecological Monographs, 72(2), pp. 203-231. https://doi.org/10.1890/00129615(2002)072[0203:IEEMOT]2.0.CO;2

9. Filatov, N.N., Druzhinin, P.V. and Menshutkin V.V., 2019. Information Support of Investigations of Environment and Socio-Economic Conditions of White Sea and Watershed. InterCarto. InterGIS. GI Support of Sustainable Development of Territories: Proceedings of the International Conference, 25(1), pp. 122-137. doi:10.35595/2414-9179-2019-1-25-122137

10. Ivanov, V.A., Igumnova, E.M. and Timchenko, I.E., 2012. Coastal Zone Resources Management. Kiev: Academperiodika, 304 p.

11. Timchenko, I.E., Igumnova, E.M. and Timchenko, I.I., 2000. [System Management and ABCTechnologies of Sustainable Development]. Sevastopol: ECOSI-Gidrofizika, 225 p. (in Russian).

12. Timchenko, I.E. and Igumnova, E.M., 2018. Biodiversity and Bio-Resource Capacity of Marine Environment in the Adaptive Model of the Ecological-Economic System Coast-Sea. Physical Oceanography, 25(3), pp. 207-219. doi:10.22449/1573-160X-2018-3-207-219

13. Timchenko, I.E., Igumnova, E.M. and Svishchev, S.V., 2019. Application of the Principles of the Marine Ecosystems' Adaptive Modeling to the Hydrochemical Observations in the Sevastopol Bay. Physical Oceanography, 26(1), pp. 63-76. doi:10.22449/1573-160X-2019-163-76

14. Timchenko, I.E., Igumnova, E.M. and Svishchev, S.V., 2020. Modeling of Intra-System Relationships in the Adaptive Model of the Marine Environment Biochemical Processes. Physical Oceanography, 27(1), pp. 81-94. doi:10.22449/1573-160X-2020-1-81-94

15. Fasham, M.J.R., Ducklow, H.W. and McKelvie, S.M., 1990. A Nitrogen-Based Model of Plankton Dynamics in the Oceanic Mixed Layer. Journal of Marine Research, 48(3), pp. 591639. https://doi.org/10.1357/002224090784984678

About the authors:

Igor. E. Timchenko, Deputy editor-in-chief of "Physical Oceanography" journal, Head of System Analysis Department, Marine Hydrophysical Institute of RAS (2 Kapitanskaya str., Sevastopol, 299011, Russian Federation), Dr. Sci. (Phys.-Math.), Professor, ResearcherID: A-86502017, ORCID ID: 0000-0002-2328-6430, timchenko.syst.analysis@mhi-ras.ru

Ekaterina M. Igumnova, Leading Engineer, Marine Hydrophysical Institute of RAS (2 Kapitanskaya str., Sevastopol, 299011, Russian Federation), SPIN-code: 5950-7785, Author ID: 861018, igumnova.syst.analysis@mhi-ras.ru

Sergey V. Svishchev, Junior Research Associate, Marine Hydrophysical Institute of RAS (2 Kapitanskaya str., Sevastopol, 299011, Russian Federation), ResearcherID: T-1962-2018, ORCID ID: 0000-0003-4673-7609, sergsvishchev09@gmail.com 
Contribution of the co-authors:

Igor E. Timchenko - research organization and its management, development and application of Adaptive Balance of Causes Method for managing scenarios of ecological and economic processes in the "coast - sea" system, analysis of research results

Ekaterina M. Igumnova - construction of a conceptual model of the ecological and economic system "coastal runoff - coastal marine environment", preparing and carrying out computational experiments with the model

Sergey V. Svishchev - development of a sea ecosystem model, construction of model equations, preparation of biochemical observation data, analysis of research results

All the authors have read and approved the final manuscript.

The authors declare that they have no conflict of interest. 